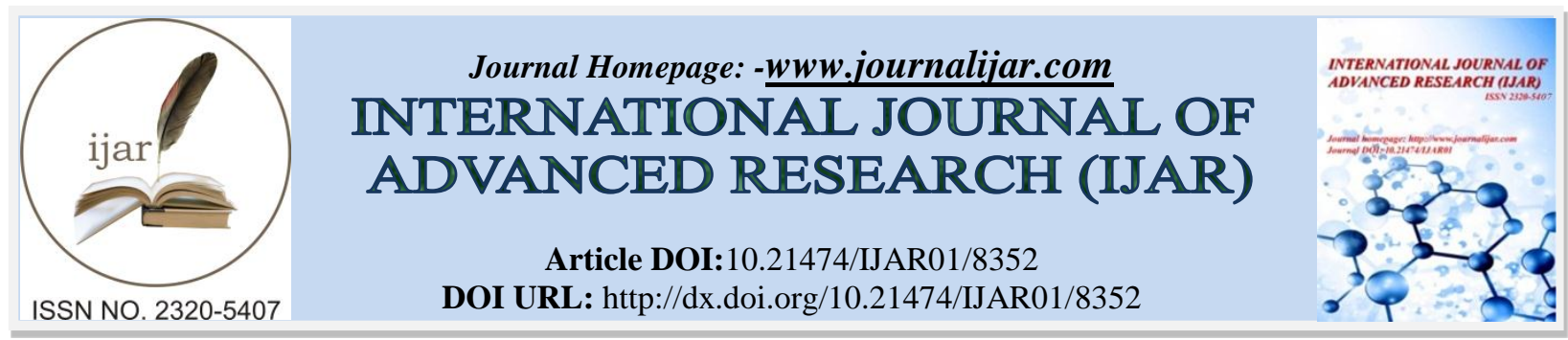

RESEARCH ARTICLE

\title{
ASSESS THE IMPACT OF CLIMATE CHANGE PARAMETERS ON RICE PRODUCTION BY VECTOR AUTOREGRESSION MODEL IN RAJSHAHI DISTRICT.
}

Md. Mostafizur Rahman and M. Sayedur Rahman.

Assistant Professor, Environmental Research Group, Department of Statistics, University of Rajshahi, Bangladesh.

\section{Manuscript Info}

\section{Manuscript History}

Received: 08 November 2018

Final Accepted: 10 December 2018

Published: January 2019

Key words:-

Aman rice, Boro rice, climate change variables, VAR model, granger causality test, Rajshahi.

\section{Abstract}

Rice is the main cereal crop of Bangladesh where three major rice crops (namely, Aus, Aman and Boro) make up the total rice production. According to World Bank report Bangladesh is treated as one of the most sensitive hotspots for climate change and climate-related extreme events. Increasing temperature and variable rainfall levels along with severe and frequent floods, droughts and cyclones adversely affect agricultural production and place Bangladesh's food security at risk. The paper examines the impact of climatic variables like rainfall, maximum temperature, minimum temperature, humidity and sunshine on two main rice crops Aman and Boro in case of Rajshahi district by Vector Autoregression (VAR) model. The empirical evidence from time series data from 1987 to 2015 confirm that all of climatic variables together influence the both rice production where rainfall and humidity has positive significant effect on Aman rice and rainfall, minimum temperature and sunshine have positive significant effect on Boro rice production in case of Rajshahi district. Therefore, it is necessary to take action to control the climatic variable to ensure food security by producing rice in large scale.

Copy Right, IJAR, 2017,. All rights reserved.

\section{Introduction:-}

The issue of climate change has become further alarming towards the healthier expansion of both socioeconomic and agriculture activities of many counties (Adejuwon, 2004). According to UNFCC report of 2007 the local climate change issue will force people's decision with penalties for their economic, personal and social environment ultimately affecting their livelihood. The scientific literature showed that high temperatures and varying rainfall intensities will be witnessed around the globe in next decade. Climate change is affecting crop yields in many low income countries where climate is a key determinant of agriculture productivity (Apata, 2010). Kurukulasuriya and Mendelsohn (2008) showed that the current global warming has turned down agriculture production, which lead to a decrease in food production.

Bangladeshi economy largely depends on agriculture where around 76 percent population is living in the rural areas and 90 percent of the rural population directly related with agriculture. The population growth rate is 2 million per year. According to this rate, the total population will become 233.2 million within 2050. However, Bangladesh faces a tremendous challenge for providing food security to the increasing population. So, it is compulsory to increase rice production in order to meet the growing demand for food emanating from population growth. The

Corresponding Author:-Md. Mostafizur Rahman.

Address:-Assistant Professor, Environmental Research Group, Department of Statistics, University of 
vulnerability of climate change on agriculture could affect the food security, trade policy, livelihood activities, etc. It can affect the crop yield positively or negatively and the changes differ largely by region and crop. Bangladesh is identified as one of the most vulnerable countries due to climate change.

The impact of climate change on agriculture production have been studied on many developing countries for example Lansigan et al., (2000); Chang, (2002); Gbetibouo and Hassan, (2005);Kurukulasuriya and Ajwad, (2007); Haimet al., (2008) and Deressa and Hassan, (2009) etc. From those studies, we found that the crop yield is more prone to climate change in developing countries. Basaket al (2010) concluded that climate change was likely to have predominately adverse impacts on the yield of Boro rice. Reilly et al. (1996) found that as temperatures move changed the production of rice. Although the effect of climate change is serious but there are limited researches conducted in case of developing countries (e.g. Boubacar, (2010); Holst et al., (2010)), and very few in case of Bangladesh (Sarker et al., (2012); Basak et al., (2010), Hossain and Silva, (2013); Hossain et al., (2012)) examined the relationship between three climatic variables (maximum temperature, minimum temperature and rainfall) and three different rice crops (Aus, Aman and Boro) in case of Bangladesh. Chowdhury and Khan (2015) examined the impact of climate change on the rice production in Bangladesh using time series data. Rahman et al (2018) examined the impact of climatic parameters on Aman rice production in case of Rangpur district, Bangladesh by using multiple regressions. The research on the effect of climatic variables on rice production is not rare in Bangladesh but findings are not converged to any unique direction due to their geographic location and different analytical methods. Rahman et al (2018) also explored the climate change effects on Boro rice yield in case of Rajshahi district by regression analysis. Janjua et. al. (2010) examined the impact of climate change on wheat production in case of Pakistan by VAR model. Farook and Kannan (2015) investigated the impact of climate change on rice production based on Vector Autoregression (VAR) model with granger causality test, impulse response and variance decomposition in case of India. Shakoor et al (2015) empirically investigated the effect of climate change on rice crop of Pakistan by employing VAR model. From the previous study we found that to investigate the impact of climatic parameters on aman and boro rice production by VAR model is rare in case of Rajshahi district.

Rice is its staple food for above 150 million Bangladeshi populations. Three major rice crops (namely, Aus, Aman and Boro) constitute $100 \%$ of total rice production and it is grown throughout the country year-round in Bangladesh. In Bangladesh, rice is grown in three seasons locally named aus (summer), aman (monsoon), andboro (winter). Summer, monsoon, and winter rice aregrown during April to August, July to December, and December to May, respectively. There are sixty four districts in Bangladesh. Rajshahi is one of the district of North West parts of Bangladesh. The historical trend for three rice production for Rajshahi district is given below:

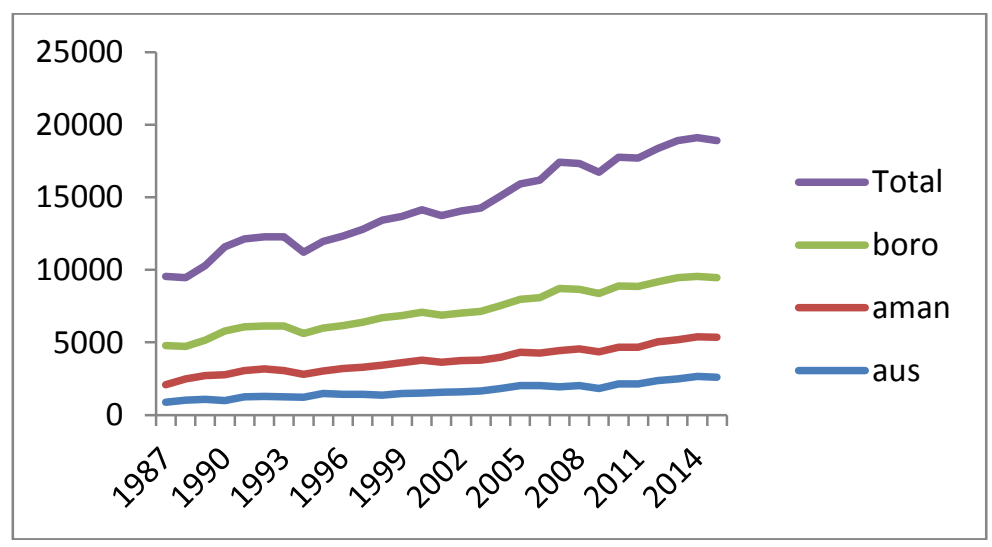

Figure 1:-Historical trend for rice production of Rajshahi, Bangladesh.

The historical trend from figure 1 indicates that Aman and Boro rice contribute the major percentage of the total rice production of Rajshahi whereas Ausrice contribute less. So, in this study we consider only Aman and Boro rice. The aim of this paper is to investigate the impact of climatic parameters on Aman and Boro rice production by Vector Autoregression model in case of Rajshahi district. The rest of the paper is organized as follows: section 2 presents the responsible parameters for climate change in Bangladesh, section 3 presents data sources and data units, section 4 presents the methodology, section 5 present result and discussion and section 6 presents the conclusion. 


\section{Responsible parameters for climate change in Bangladesh:-}

It is now widely recognized that Bangladesh is considered as one of the most sensitive hotspots for climate change and climate-related extreme events (World Bank, 2013). Increasing temperature and variable rainfall levels along with severe and frequent floods, droughts and cyclones adversely affect agricultural production and place Bangladesh's food security at risk $(\mathrm{GoB}, 2011)$.The responsible parameters for climate change in Bangladesh areRainfall, Temperature (Maximum Temperature, Minimum temperature), Sunshine and Humidity. So in our study we consider rainfall, maximum temperature, minimum temperature, humidity and sunshine as climatic variables.

\section{Data Source and Data Units:-}

The study was conducted in a purposely-selected Rajshahi district.The study was limited to some climatic factors. The selectedclimatic variables are maximum temperature, minimum temperature, total rainfall, sunshine hours and humidity.The monthly maximum temperature, minimum temperature and total rainfall data for months from January to December for the period 1985-2015 were obtained from Bangladesh Meteorological Department (BMD), Bangladesh Bureau of Statistics (BBS) and theDepartment of Agricultural Extension (DAE). Rice production data for particular district was collected from the Year book of Agricultural Statistics of Bangladesh of BBS. Data also collected from other relevant resources.

Data has been entered and processed in the computer by using MS-Excel program. All data were converted into standard units such as yield is converted into $\mathrm{kg} /$ hectare, rainfall into $\mathrm{mm}$, temperature into ${ }^{\circ} \mathrm{C}$, sunshine into hours and humidity into percentage. All of these data also converted for Aman rice from July to December and for Boro rice from December to May.

\section{Methodology:-}

Generally, the structural approach to time series modeling uses economic theory to model the relationshipamong different variables. But sometimes economic theory is often not wealthy enough to identify all of these relationships. Furthermore, estimation and inference are complicated by the fact that endogenous variables mayappear on both the left and right sides of equations. These problems lead to consider non-structural approaches to modeling the relationshipamong several variables. Sims (1980) introduced the VAR model. The vector autoregression (VAR) is commonly used for forecasting systems of interrelated time series and for analyzing the dynamic impact of random disturbances on the system of variables. The VAR approach sidesteps the need for structural modeling by treating every endogenous variable in the system as a function of the lagged values of all of the endogenous variables in the system. VAR models are the multivariate extensions of the univariate AR models to the multivariate case and they explain and/or predict the values of a set of variables at any given point in time (Sims, (1980); Todd, (1984) and Farook et al (2015)). The mathematical representation of VAR is given below:

$y_{t}=\beta_{1} y_{t-1}+\ldots+\beta_{p} y_{t-p}+\delta x_{t}+\varepsilon_{t}$

Where $y_{t}$ is a $\mathrm{k}$ vector of endogenous variables, $x_{t}$ is a $\mathrm{d}$ vector of exogenous variables, $\beta_{1}, \ldots, \beta_{p}$ and $\delta$ are matrices of coefficient to be estimated, and $\varepsilon_{t}$ is a vector of innovations that may be contemporaneously correlated but are uncorrelated with their own lagged values and uncorrelated with all of the right hand side variables. General equation including climatic variables and non-climatic variables of the study is:

Rice production $=f$ (rainfall, max. temperature, min. temperature, humidity sunshine, $)$.

Therefore, the econometric form of the model can be written as

$$
P D=\beta_{1}+\beta_{2} R A+\beta_{3} T \max +\beta_{4} T \min +\beta_{5} H U+\beta_{6} S S+\varepsilon_{t}
$$

Where PD indicates yield (in $\mathrm{kg}$ per hectare), RA indicates rainfall, Tmaxindicates maximum temperature, Tmin indicates minimum temperature, HU indicates humidity and SS indicates sunshine for both rice.

\section{Augmented Dickey-Fuller test:-}

Prior to run the VAR model we need to confirm the non-stationary property of these series. It is important to make sure that all of the variables are stationary, because if they are non-stationary then the standard assumption for asymptotic analysis in the Granger test will not valid. There are several methods to test the stationarity of the time series data such as Augmented Dickey-Fuller (Dickey-Fuller 1979), Dickey-Fuller (1981), Phillips-Perron (1988). In our study we only consider Augmented Dickey-Fuller test. The test statistic is given below:

$$
\Delta X_{t}=\mu+(\rho-1) X_{t-1}+\eta T+\xi \Delta X_{t-1}+u_{2 t}
$$




\section{Granger Causality test:-}

According to Granger (1969) the question of whether $X$ causes $Y$ is to see how much of the current $Y$ can be explained by past values of $Y$ and then to see whether adding lagged values of $X$ can improve the explanation. $Y$ is said to be granger-caused by $X$ if $X$ helps in the prediction of $Y$, or equivalently if the co-efficient on the lagged $X$ 's are statistically significant. Granger causality distinguishes between unidirectional and bi-directional causality. Unidirectional causality is said to exist from $X$ to $Y$ if $X$ causes $Y$ but $Y$ does not causes $X$. In order to test for Granger causality, we will estimate a VAR model as follows, where all variables are initially considered symmetrically and endogenously.

$$
\begin{gathered}
{\left[L X_{t}\right]=\alpha+\beta_{1}\left[L X_{t-1}\right]+\beta_{2}\left[L X_{t-2}\right]+\ldots+\beta_{n}\left[L X_{t-n}\right]+\left[U_{t}\right]} \\
X=\left[\begin{array}{llllll}
P D & R A & T \max & T \min & H U & S S
\end{array}\right]^{\prime}
\end{gathered}
$$

\section{Results and Discussions:-}

The summary statistics such as mean, standard deviation, minimum, maximum values, skewness, kurtosis of these series are given at Table-1. These statistics indicate that mean yield of Aman rice is less than mean yield of Boro rice. The highest mean maximum and minimum temperature, rainfall is observed for Aman rice where highest mean humidity and sunshine is observed for Boro rice. All of the series for both Aman and Boro rice are not normally distributed. This table also indicates that all of the series for both rice are negatively skewed except the series rainfall, maximum temperature and minimum temperature for Boro rice.

\begin{tabular}{|c|c|c|c|c|c|c|c|c|c|c|c|c|}
\hline Statistics & \multicolumn{6}{|c|}{ Aman } & \multicolumn{6}{|c|}{ Boro } \\
\hline & Yield & RA & Tmax & Tmin & HU & SS & Yield & $\mathbf{R A}$ & Tmax & Tmin & HU & SS \\
\hline Mean & $\begin{array}{l}2113 . \\
2\end{array}$ & $\begin{array}{l}6.194 \\
6\end{array}$ & $\begin{array}{l}33.91 \\
7\end{array}$ & $\begin{array}{l}24.52 \\
4\end{array}$ & $\begin{array}{l}78.97 \\
0\end{array}$ & 5.636 & $\begin{array}{l}3430 . \\
2\end{array}$ & $\begin{array}{l}4.274 \\
3\end{array}$ & $\begin{array}{l}30.50 \\
8\end{array}$ & $\begin{array}{l}21.26 \\
2\end{array}$ & $\begin{array}{l}80.90 \\
2\end{array}$ & $\begin{array}{l}6.11 \\
3\end{array}$ \\
\hline Median & $\begin{array}{l}2123 . \\
8\end{array}$ & $\begin{array}{l}6.001 \\
0\end{array}$ & $\begin{array}{l}34.14 \\
7\end{array}$ & $\begin{array}{l}25.04 \\
2\end{array}$ & $\begin{array}{l}79.31 \\
4\end{array}$ & 5.935 & $\begin{array}{l}3293 . \\
9\end{array}$ & 4.079 & $\begin{array}{l}30.48 \\
7\end{array}$ & $\begin{array}{l}21.07 \\
2\end{array}$ & $\begin{array}{l}83.01 \\
4\end{array}$ & $\begin{array}{l}6.47 \\
6\end{array}$ \\
\hline $\begin{array}{l}\text { Maximu } \\
\mathrm{m}\end{array}$ & $\begin{array}{l}2748 . \\
4\end{array}$ & $\begin{array}{l}10.27 \\
0\end{array}$ & $\begin{array}{l}35.55 \\
0\end{array}$ & $\begin{array}{l}26.07 \\
1\end{array}$ & $\begin{array}{l}83.48 \\
2\end{array}$ & 7.277 & $\begin{array}{l}4277 . \\
6\end{array}$ & 6.637 & $\begin{array}{l}31.40 \\
5\end{array}$ & $\begin{array}{l}30.11 \\
5\end{array}$ & $\begin{array}{l}84.75 \\
3\end{array}$ & $\begin{array}{l}7.33 \\
6\end{array}$ \\
\hline $\begin{array}{l}\text { Minimu } \\
\mathrm{m}\end{array}$ & $\begin{array}{l}1212 . \\
3\end{array}$ & $\begin{array}{l}1.504 \\
5\end{array}$ & $\begin{array}{l}30.66 \\
7\end{array}$ & $\begin{array}{l}17.96 \\
0\end{array}$ & $\begin{array}{l}70.40 \\
7\end{array}$ & 1.00 & $\begin{array}{l}2253 . \\
3\end{array}$ & 2.240 & $\begin{array}{l}29.67 \\
3\end{array}$ & $\begin{array}{l}19.89 \\
4\end{array}$ & $\begin{array}{l}30.11 \\
5\end{array}$ & $\begin{array}{l}1.00 \\
0\end{array}$ \\
\hline Std & $\begin{array}{l}416.8 \\
3\end{array}$ & $\begin{array}{l}1.886 \\
6\end{array}$ & $\begin{array}{l}1.138 \\
7\end{array}$ & $\begin{array}{l}1.836 \\
6\end{array}$ & 3.119 & 1.511 & $\begin{array}{l}594.1 \\
0\end{array}$ & 1.085 & 0.360 & 1.790 & $\begin{array}{l}10.03 \\
7\end{array}$ & $\begin{array}{l}1.48 \\
3\end{array}$ \\
\hline $\begin{array}{l}\text { Skewnes } \\
\text { s }\end{array}$ & $-\overline{0.205}$ & $-\overline{0.105}$ & $-\overline{0.968}$ & $\overline{0} 0.522$ & $-\overline{0.253}$ & $-\overline{0.227}$ & $-\overline{0.012}$ & 0.459 & 0.092 & 0.341 & $\overline{0}-\overline{710}$ & 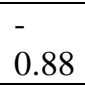 \\
\hline Kurtosis & 2.156 & $\begin{array}{l}2.035 \\
1\end{array}$ & $\begin{array}{l}2.901 \\
7\end{array}$ & $\begin{array}{l}2.384 \\
7\end{array}$ & $\begin{array}{l}2.624 \\
0\end{array}$ & $\begin{array}{l}2.136 \\
2\end{array}$ & 1.933 & 2.870 & 2.273 & 2.159 & $\begin{array}{l}2.263 \\
9\end{array}$ & $\begin{array}{l}1.45 \\
6\end{array}$ \\
\hline $\begin{array}{l}\text { J-B test } \\
\text { (Pvalue) }\end{array}$ & $\begin{array}{l}0.587 \\
5\end{array}$ & .8725 & $\begin{array}{l}0.634 \\
1\end{array}$ & $\begin{array}{l}0.234 \\
1\end{array}$ & $\begin{array}{l}0.455 \\
0\end{array}$ & $\begin{array}{l}0.213 \\
4\end{array}$ & $\begin{array}{l}0.502 \\
1\end{array}$ & $\begin{array}{l}0.594 \\
1\end{array}$ & 0.946 & $\begin{array}{l}0.021 \\
2\end{array}$ & 0.312 & $\begin{array}{l}0.41 \\
2\end{array}$ \\
\hline
\end{tabular}

Table 1:-Summary statistics for Aman and Boro rice

Vector Auto Regression (VAR) requires the testing the stationarity ofthese variables. The estimation results for unit root test statistics are done for both trend and without trend for levels and also for their first differences. These results are given at Table- 2 .

Table 2:-Estimation results ofunit root test for both rice.

\begin{tabular}{|l|l|l|l|l|l|c|c|c|}
\hline \multirow{3}{*}{ Variables } & \multicolumn{4}{|c|}{ Aman } & \multicolumn{4}{c|}{ Boro } \\
\cline { 2 - 9 } & \multicolumn{2}{|c|}{ ADF(level) } & \multicolumn{2}{c|}{ ADF (first diff) } & \multicolumn{2}{c|}{ ADF (Level) } & \multicolumn{2}{|c|}{ ADF(first diff.) } \\
\cline { 2 - 9 } & $\begin{array}{c}\text { Without } \\
\text { trend }\end{array}$ & $\begin{array}{c}\text { With } \\
\text { trend }\end{array}$ & $\begin{array}{c}\text { Without } \\
\text { trend }\end{array}$ & $\begin{array}{c}\text { With } \\
\text { trend }\end{array}$ & $\begin{array}{c}\text { Without } \\
\text { trend }\end{array}$ & $\begin{array}{c}\text { With } \\
\text { trend }\end{array}$ & $\begin{array}{c}\text { Without } \\
\text { trend }\end{array}$ & $\begin{array}{c}\text { With } \\
\text { trend }\end{array}$ \\
\hline Yield & 5.156 & -5.713 & -4.482 & -4.587 & -0.945 & -3.176 & -6.306 & -6.301 \\
\hline RA & -1.087 & -6.551 & -5.558 & -5.475 & -0.052 & -4.098 & -9.446 & -9.088 \\
\hline Tmax & -0.058 & -2.143 & -6.913 & -6.424 & 0.0753 & -6.118 & -9.797 & -7.194 \\
\hline Tmin & 0.0289 & -6.238 & -8.375 & -7.911 & 0.826 & -2.094 & -2.244 & -5.447 \\
\hline HU & -0.951 & -0.531 & -2.883 & -4.523 & -1.059 & 1.409 & -5.131 & -4.445 \\
\hline SS & -0.156 & -5.021 & -8.205 & -7.922 & -0.577 & -3.088 & -5.576 & -5.381 \\
\hline
\end{tabular}


From Table-2, we found that the null hypothesis of nonstationarity cannot be rejected for some series for both Aman and Boro rice which indicates that all of these series are non stationary both at $5 \%$ and also $10 \%$ significance but some series for both rice shown stationarity at their level for both trend and without trend. Then we consider first difference and found that the null hypothesis can be rejected which indicates that all of these series are stationary at $5 \%$ as well as $10 \%$ level of significance. So finally we found the stationarity of these series at their first differences. Johansen's procedure of multivariate cointegration requires the existence of sufficient number of time lags. Akaike Information Criterion with critical values from Osterwald-Lenum (1992) indicates the lag length is 2 for both Aman and Boro rice.

Table 3:-VAR estimation results for Aman and Boro rice.

\begin{tabular}{|c|c|c|c|c|c|c|c|c|c|c|c|c|}
\hline & \multicolumn{6}{|c|}{ Aman } & \multicolumn{6}{|c|}{ Boro } \\
\hline & Yield & RA & Tmax & $\underset{\mathbf{n}}{\mathbf{T}}$ & HU & SS & Yield & RA & Tmax & Tmin & HU & SS \\
\hline $\begin{array}{l}\text { Yi(- } \\
\text { 1) }\end{array}$ & $\begin{array}{c}1.004 \\
(0.26 \\
3) \\
{[} \\
3.812]\end{array}$ & $\begin{array}{c}0.261 \\
(0.02 \\
4) \\
{[} \\
2.467]\end{array}$ & $\begin{array}{c}-0.001 \\
(0.00 \\
2) \\
{[-} \\
0.771]\end{array}$ & $\begin{array}{c}0.00 \\
2 \\
(0.0 \\
0) \\
{[} \\
0.06]\end{array}$ & $\begin{array}{c}0.20 \\
5 \\
(0.1 \\
3) \\
{[} \\
2.39]\end{array}$ & $\begin{array}{c}0.00 \\
5 \\
(0.0 \\
0) \\
{[} \\
0.19]\end{array}$ & $\begin{array}{c}0.925 \\
(0.17 \\
8) \\
{[5.19]}\end{array}$ & $\begin{array}{c}0.511 \\
(0.11 \\
7) \\
{[2.08]}\end{array}$ & $\begin{array}{c}0.001 \\
(0.10 \\
4) \\
{[0.43]}\end{array}$ & $\begin{array}{c}0.211 \\
(0.00 \\
1) \\
{[1.99]}\end{array}$ & $\begin{array}{c}- \\
0.004 \\
(0.0 \\
6) \\
{[-} \\
0.79]\end{array}$ & $\begin{array}{c}0.120 \\
(0.11 \\
2) \\
{[1.534} \\
]\end{array}$ \\
\hline $\begin{array}{l}\text { Yi(- } \\
\text { 2) }\end{array}$ & $\begin{array}{c}-0.064 \\
(0.27 \\
8) \\
{[-} \\
0.230]\end{array}$ & $\begin{array}{c}0.380 \\
(0.10 \\
4) \\
{[2.884} \\
]\end{array}$ & $\begin{array}{c}0.001 \\
(0.00 \\
2) \\
{[} \\
0.674]\end{array}$ & $\begin{array}{c}- \\
0.002 \\
(0.0 \\
4) \\
{[-} \\
0.51]\end{array}$ & $\begin{array}{c}0.111 \\
(0.1 \\
3) \\
{[1.87} \\
]\end{array}$ & $\begin{array}{c}- \\
0.001 \\
(0.0 \\
3) \\
{[-} \\
0.46]\end{array}$ & $\begin{array}{c}-0.062 \\
(0.18) \\
{[-0.34]}\end{array}$ & $\begin{array}{c}0.203 \\
(0.01 \\
2) \\
{[2.32]}\end{array}$ & $\begin{array}{c}0.110 \\
(0.04 \\
1) \\
{[0.79]}\end{array}$ & $\begin{array}{c}0.304 \\
(0.00 \\
1) \\
{[2.13]}\end{array}$ & $\begin{array}{c}- \\
0.002 \\
(0.0 \\
2) \\
{[-} \\
0.47] \\
\end{array}$ & $\begin{array}{c}0.108 \\
(0.07 \\
8) \\
{[} \\
2.463]\end{array}$ \\
\hline $\begin{array}{l}\text { RA(- } \\
\text { 1) }\end{array}$ & $\begin{array}{c}54.76 \\
(25.6 \\
8) \\
{[} \\
2.132]\end{array}$ & $\begin{array}{c}-0.403 \\
(0.40 \\
7) \\
{[-0.99]}\end{array}$ & $\begin{array}{c}-0.073 \\
(0.20 \\
2) \\
{[-0.36]}\end{array}$ & $\begin{array}{c}- \\
0.076 \\
(0.3 \\
8) \\
{[-} \\
0.19]\end{array}$ & $\begin{array}{c}-1.06 \\
(1.2 \\
6) \\
{[-} \\
0.84]\end{array}$ & $\begin{array}{c}0.23 \\
4 \\
(0.2 \\
9) \\
{[} \\
0.73] \\
\end{array}$ & $\begin{array}{r}100.52 \\
(39.7) \\
{[2.53]}\end{array}$ & $\begin{array}{l}0.076 \\
(0.28) \\
{[0.26]}\end{array}$ & $\begin{array}{c}0.016 \\
(0.08) \\
{[0.18]}\end{array}$ & $\begin{array}{c}0.151 \\
(0.26) \\
{[0.56]}\end{array}$ & $\begin{array}{c}- \\
2.907 \\
(1.3 \\
5) \\
{[-} \\
2.12] \\
\end{array}$ & $\begin{array}{c}0.868 \\
(0.38) \\
{[2.24]}\end{array}$ \\
\hline $\begin{array}{l}\text { RA(- } \\
\text { 2) }\end{array}$ & $\begin{array}{c}9.282 \\
(26.1 \\
9) \\
{[} \\
0.354]\end{array}$ & $\begin{array}{c}0.557 \\
(0.41 \\
5) \\
{[1.341} \\
]\end{array}$ & $\begin{array}{c}0.057 \\
(0.20 \\
6) \\
{[} \\
0.285]\end{array}$ & $\begin{array}{c}- \\
0.871 \\
(0.3 \\
9) \\
{[-} \\
2.23] \\
\end{array}$ & $\begin{array}{c}- \\
0.657 \\
(1.2 \\
8) \\
{[-} \\
0.51]\end{array}$ & $\begin{array}{c}0.02 \\
4 \\
(0.3 \\
0) \\
{[} \\
0.08] \\
\end{array}$ & $\begin{array}{c}6.90 \\
(41.5 \\
2) \\
{[0.16]}\end{array}$ & $\begin{array}{c}-0.121 \\
(0.29) \\
{[-} \\
0.409]\end{array}$ & $\begin{array}{c}0.145 \\
(0.09 \\
2) \\
{[1.57]}\end{array}$ & $\begin{array}{c}0.738 \\
(0.27) \\
{[2.66]}\end{array}$ & $\begin{array}{c}- \\
2.275 \\
(1.4 \\
1) \\
{[-} \\
1.61]\end{array}$ & $\begin{array}{r}-0.225 \\
(0.41) \\
{[-0.55]}\end{array}$ \\
\hline $\begin{array}{l}\text { Tmax } \\
(-1)\end{array}$ & $\begin{array}{c}-68.86 \\
(41.1 \\
3) \\
{[-1.67]}\end{array}$ & $\begin{array}{r}-0.844 \\
(0.65) \\
{[-1.29]}\end{array}$ & $\begin{array}{c}-0.14 \\
(0.32) \\
{[-} \\
0.447]\end{array}$ & $\begin{array}{c}1.18 \\
(0.6 \\
1) \\
{[} \\
1.93]\end{array}$ & $\begin{array}{c}- \\
10.33 \\
(2.0 \\
2) \\
{[-} \\
5.11]\end{array}$ & $\begin{array}{c}0.55 \\
(0.4 \\
7) \\
{[} \\
1.18]\end{array}$ & $\begin{array}{l}295.7 \\
(135 .) \\
{[2.12]}\end{array}$ & $\begin{array}{c}-1.36 \\
(0.96) \\
{[-1.41]}\end{array}$ & $\begin{array}{c}0.09 \\
(0.30) \\
{[0.32]}\end{array}$ & $\begin{array}{c}-0.50 \\
(0.90) \\
{[-0.55]}\end{array}$ & $\begin{array}{r}-8.80 \\
(4.6) \\
{[-1.9]}\end{array}$ & $\begin{array}{c}2.59 \\
(1.32) \\
{[1.96]}\end{array}$ \\
\hline $\begin{array}{c}\text { Tmax } \\
(-2)\end{array}$ & $\begin{array}{c}50.71 \\
(53.2 \\
0) \\
{[0.95]}\end{array}$ & $\begin{array}{c}0.074 \\
(0.84 \\
3) \\
{[} \\
0.087]\end{array}$ & $\begin{array}{c}-0.144 \\
(0.41 \\
8) \\
{[-} \\
0.344]\end{array}$ & $\begin{array}{c}- \\
1.226 \\
(0.7 \\
9) \\
{[-} \\
1.54]\end{array}$ & $\begin{array}{c}- \\
1.732 \\
(2.6 \\
1) \\
{[-} \\
0.60]\end{array}$ & $\begin{array}{c}0.42 \\
6 \\
(0.6 \\
1) \\
{[} \\
0.69]\end{array}$ & $\begin{array}{c}103.8 \\
(136) \\
{[1.76]}\end{array}$ & $\begin{array}{c}-1.24 \\
(0.97) \\
{[-1.28]}\end{array}$ & $\begin{array}{c}0.21 \\
(0.30) \\
{[0.70]}\end{array}$ & $\begin{array}{c}2.06 \\
(0.91) \\
{[2.26]}\end{array}$ & $\begin{array}{c}- \\
10.26 \\
(4.6) \\
{[-2.2]}\end{array}$ & $\begin{array}{c}0.85 \\
(1.32) \\
{[0.64]}\end{array}$ \\
\hline $\begin{array}{l}\text { Tmin } \\
(-1)\end{array}$ & $\begin{array}{c}16.56 \\
(24.9 \\
7) \\
{[} \\
0.663]\end{array}$ & $\begin{array}{c}0.145 \\
(0.39 \\
5) \\
{[} \\
0.368]\end{array}$ & $\begin{array}{c}0.462 \\
(0.19) \\
{[2.35]}\end{array}$ & $\begin{array}{c}- \\
1.378 \\
(0.3 \\
7) \\
{[-} \\
3.76]\end{array}$ & $\begin{array}{c}6.31 \\
(1.2 \\
2) \\
{[} \\
5.13]\end{array}$ & $\begin{array}{c}- \\
0.241 \\
(0.2 \\
8) \\
{[-} \\
0.84]\end{array}$ & $\begin{array}{c}-32.61 \\
(71.3) \\
{[-0.45]}\end{array}$ & $\begin{array}{c}0.47 \\
(0.50 \\
8) \\
{[0.92]}\end{array}$ & $\begin{array}{c}0.24 \\
(0.15 \\
8) \\
{[2.30]}\end{array}$ & $\begin{array}{c}0.73 \\
(0.47) \\
{[} \\
1.535]\end{array}$ & $\begin{array}{c}0.56 \\
(2.4 \\
2) \\
{[} \\
0.23]\end{array}$ & $\begin{array}{c}-0.39 \\
(0.69) \\
{[-0.56]}\end{array}$ \\
\hline
\end{tabular}




\begin{tabular}{|c|c|c|c|c|c|c|c|c|c|c|c|c|}
\hline $\begin{array}{l}\text { Tmin } \\
(-2)\end{array}$ & $\begin{array}{c}4.612 \\
(40.0 \\
2) \\
{[} \\
0.115]\end{array}$ & $\begin{array}{c}0.303 \\
(0.63 \\
4) \\
{[} \\
0.478]\end{array}$ & $\begin{array}{c}-0.066 \\
(0.31 \\
4) \\
{[-} \\
0.212]\end{array}$ & $\begin{array}{c}- \\
0.026 \\
(0.5 \\
9) \\
{[-} \\
0.04]\end{array}$ & $\begin{array}{c}4.43 \\
1 \\
(1.9 \\
6) \\
{[} \\
2.25]\end{array}$ & $\begin{array}{c}0.08 \\
1 \\
(0.4 \\
5) \\
{[} \\
0.17]\end{array}$ & $\begin{array}{c}160.6 \\
(73.5 \\
7) \\
{[} \\
2.182]\end{array}$ & $\begin{array}{c}0.89 \\
(0.52) \\
{[} \\
1.704]\end{array}$ & $\begin{array}{c}0.21 \\
(0.16 \\
3) \\
{[1.89]}\end{array}$ & $\begin{array}{c}0.67 \\
(0.49 \\
1) \\
{[1.37]}\end{array}$ & $\begin{array}{c}4.89 \\
(2.5 \\
0) \\
{[} \\
1.91]\end{array}$ & $\begin{array}{c}-0.439 \\
(0.71 \\
5) \\
{[-} \\
0.614]\end{array}$ \\
\hline $\begin{array}{l}\mathrm{HU}(- \\
1)\end{array}$ & $\begin{array}{c}4.612 \\
(40.0 \\
2) \\
{[} \\
0.115]\end{array}$ & $\begin{array}{c}0.303 \\
(0.63 \\
4) \\
{[} \\
0.478]\end{array}$ & $\begin{array}{c}-0.066 \\
(0.31 \\
4) \\
{[-} \\
0.212]\end{array}$ & $\begin{array}{c}- \\
0.026 \\
(0.5 \\
9) \\
{[-} \\
0.04]\end{array}$ & $\begin{array}{c}4.43 \\
(1.9 \\
6) \\
{[} \\
2.29]\end{array}$ & $\begin{array}{c}0.08 \\
1 \\
(0.4 \\
5) \\
{[} \\
0.17]\end{array}$ & $\begin{array}{c}-16.71 \\
(16.9 \\
5) \\
{[-0.98]}\end{array}$ & $\begin{array}{c}-0.352 \\
(0.12) \\
{[-2.91]}\end{array}$ & $\begin{array}{c}0.062 \\
(0.03) \\
{[1.65]}\end{array}$ & $\begin{array}{r}-0.662 \\
(0.11) \\
{[-5.84]}\end{array}$ & $\begin{array}{c}2.98 \\
6 \\
(0.5 \\
7) \\
{[} \\
5.17]\end{array}$ & $\begin{array}{c}0.131 \\
(0.16 \\
4) \\
{[} \\
0.797]\end{array}$ \\
\hline $\begin{array}{l}\text { HU(- } \\
\text { 2) }\end{array}$ & $\begin{array}{c}53.35 \\
(23.1 \\
5) \\
{[2.30]}\end{array}$ & $\begin{array}{c}-0.111 \\
(0.36 \\
6) \\
{[-} \\
0.305]\end{array}$ & $\begin{array}{c}0.066 \\
(0.18) \\
{[} \\
0.332]\end{array}$ & $\begin{array}{c}0.65 \\
1 \\
(0.3 \\
4) \\
{[} \\
1.91]\end{array}$ & $\begin{array}{c}2.203 \\
(1.1 \\
3) \\
{[1.94} \\
]\end{array}$ & $\begin{array}{c}0.28 \\
1 \\
(0.2 \\
6) \\
{[} \\
1.06]\end{array}$ & $\begin{array}{c}6.17 \\
(29.0 \\
9) \\
{[} \\
0.212]\end{array}$ & $\begin{array}{c}0.039 \\
(0.20) \\
{[} \\
0.191]\end{array}$ & $\begin{array}{r}-0.016 \\
(0.06) \\
{[-0.25]}\end{array}$ & $\begin{array}{c}-0.134 \\
(0.19 \\
4) \\
{[-0.69]}\end{array}$ & $\begin{array}{c}2.49 \\
(0.9 \\
8) \\
{[} \\
2.02]\end{array}$ & $\begin{array}{c}-0.051 \\
(0.28 \\
3) \\
{[-} \\
0.187]\end{array}$ \\
\hline $\begin{array}{l}\text { SS } \\
(-1)\end{array}$ & $\begin{array}{c}34.78 \\
(21.8 \\
2) \\
{[1.59]}\end{array}$ & $\begin{array}{c}0.481 \\
(0.34 \\
6) \\
{[1.39]}\end{array}$ & $\begin{array}{c}-0.138 \\
(0.17 \\
1) \\
{[-} \\
0.806]\end{array}$ & $\begin{array}{c}0.25 \\
0 \\
(0.3 \\
2) \\
{[} \\
0.77]\end{array}$ & $\begin{array}{c}0.57 \\
8 \\
(1.0 \\
7) \\
{[} \\
0.53]\end{array}$ & $\begin{array}{c}0.24 \\
6 \\
(0.2 \\
5) \\
{[} \\
0.98]\end{array}$ & $\begin{array}{c}66.81 \\
(27.2 \\
9) \\
{[2.44]}\end{array}$ & $\begin{array}{c}0.29 \\
(0.19) \\
{[1.49]}\end{array}$ & $\begin{array}{r}-0.192 \\
(0.06) \\
{[-3.17]}\end{array}$ & $\begin{array}{c}0.02 \\
(0.18 \\
2) \\
{[0.15]}\end{array}$ & $\begin{array}{c}- \\
1.025 \\
(0.9 \\
2) \\
{[-} \\
1.10]\end{array}$ & $\begin{array}{c}0.730 \\
(0.26) \\
{[} \\
2.752]\end{array}$ \\
\hline $\begin{array}{l}S S \\
(-2)\end{array}$ & $\begin{array}{c}-51.04 \\
(28.9 \\
4) \\
{[-1.76]}\end{array}$ & $\begin{array}{r}-0.431 \\
(0.45) \\
{[-0.93]}\end{array}$ & $\begin{array}{c}0.277 \\
(0.22 \\
8) \\
{[1.21]}\end{array}$ & $\begin{array}{c}- \\
0.029 \\
(0.4 \\
3) \\
{[-} \\
0.06]\end{array}$ & $\begin{array}{c}- \\
1.598 \\
(1.4 \\
2) \\
{[-} \\
1.11]\end{array}$ & $\begin{array}{c}- \\
0.347 \\
(0.3 \\
3) \\
{[-} \\
1.04]\end{array}$ & $\begin{array}{c}29.36 \\
(31.3 \\
0) \\
{[0.93]}\end{array}$ & $\begin{array}{c}-0.21 \\
(0.22) \\
{[-0.97]}\end{array}$ & $\begin{array}{c}0.088 \\
(0.06) \\
{[1.26]}\end{array}$ & $\begin{array}{c}0.28 \\
(0.20) \\
{[1.38]}\end{array}$ & $\begin{array}{c}2.39 \\
(1.0 \\
6) \\
{[-} \\
2.24]\end{array}$ & $\begin{array}{c}0.171 \\
(0.30) \\
{[0.562} \\
]\end{array}$ \\
\hline $\mathrm{c}$ & $\begin{array}{c}1600 . \\
6 \\
(2279 \\
) \\
{[} \\
0.702]\end{array}$ & $\begin{array}{c}2.444 \\
(36.1 \\
2) \\
{[} \\
0.067]\end{array}$ & $\begin{array}{c}40.41 \\
(17.9 \\
3) \\
{[2.25]}\end{array}$ & $\begin{array}{c}0.90 \\
4 \\
(33 . \\
9) \\
{[} \\
0.02]\end{array}$ & $\begin{array}{c}373 \\
(111 \\
.) \\
{[} \\
3.33]\end{array}$ & $\begin{array}{c}- \\
64.11 \\
(26 . \\
1) \\
{[-} \\
2.44]\end{array}$ & $\begin{array}{c}11536 \\
. \\
(7505 \\
.) \\
{[1.53]}\end{array}$ & $\begin{array}{c}82.73 \\
(53.4 \\
9) \\
{[1.54]}\end{array}$ & $\begin{array}{c}28.19 \\
(16.6 \\
9) \\
{[1.63]}\end{array}$ & $\begin{array}{c}29.74 \\
(50.1 \\
5) \\
{[0.59]}\end{array}$ & $\begin{array}{c}204 . \\
3 \\
(255 \\
) \\
{[} \\
0.81]\end{array}$ & $\begin{array}{c}- \\
94.045 \\
(73.0 \\
1) \\
{[-} \\
1.288]\end{array}$ \\
\hline R.sq & 0.896 & -0.044 & 0.374 & $\begin{array}{c}0.28 \\
2\end{array}$ & $\begin{array}{c}0.64 \\
4\end{array}$ & $\begin{array}{c}0.26 \\
1\end{array}$ & 0.961 & 0.538 & 0.570 & 0.851 & $\begin{array}{c}0.87 \\
7\end{array}$ & 0.535 \\
\hline $\begin{array}{l}\text { Adj.R } \\
\text { s }\end{array}$ & 0.866 & -0.190 & 0.285 & 0.350 & 0.681 & $\begin{array}{c}0.39 \\
7\end{array}$ & 0.929 & 0.142 & 0.201 & 0.723 & $\begin{array}{c}0.77 \\
2\end{array}$ & 0.138 \\
\hline ssr & $\begin{array}{c}118.4 \\
8\end{array}$ & 1.878 & 0.932 & $\begin{array}{c}1.76 \\
5\end{array}$ & $\begin{array}{c}5.81 \\
2\end{array}$ & $\begin{array}{c}1.36 \\
1\end{array}$ & 822.4 & 15.18 & 1.478 & 13.34 & $\begin{array}{c}345 . \\
4\end{array}$ & 28.28 \\
\hline S.eeq & 19.74 & 0.90 & 2.294 & $\begin{array}{c}1.85 \\
2\end{array}$ & $\begin{array}{c}4.92 \\
3\end{array}$ & $\begin{array}{c}1.76 \\
7\end{array}$ & 146.9 & 1.041 & 0.324 & 0.977 & $\begin{array}{c}4.96 \\
7\end{array}$ & 1.421 \\
\hline F-Stat & $\begin{array}{c}- \\
158.36\end{array}$ & -46.46 & -27.54 & -44.7 & -76.9 & -37.7 & 29.35 & 1.359 & 1.54 & 6.677 & 8.35 & 1.34 \\
\hline $\mathrm{LL}$ & 0.896 & -0.044 & 0.374 & $\begin{array}{c}0.28 \\
2\end{array}$ & $\begin{array}{c}0.64 \\
4\end{array}$ & $\begin{array}{c}0.26 \\
1\end{array}$ & -164.0 & -30.53 & 0.906 & -28.79 & -72.7 & -38.93 \\
\hline
\end{tabular}

From Table-3, the results of VAR model estimation revealed that though t statistics of some variables are significant while some are non significantfor both Aman and Boro rice at conventional level of significance however F-statistic value for the both Aman and Boro rice is very high and also statistically significant so the model is said to a best fit and this higher value has made all the lag terms significant. The coefficient of determination R-square for Aman is 0.896 and for Boro is 0.961 and the adjusted coefficient of determination R-square are 0.866 and 0.929 for Aman and Boro respectively. Both the values are lying between 0 and 1 and also both the values are very high which also 
shows the goodness of the fit of the overall model. Therefore we can say $89.6 \%$ variation can be explained by the climatic variables for aman rice and $96.1 \%$ variation can be explained by climatic variables for boro rice. So, the impacts of climatic variables are significant for both Aman and Boro rice.

\section{VAR Granger Causality/Block Exogeneity Wald Tests:-}

Under this system, an endogenous variable can be treated as exogenous. We used the Chi-square (Wald) test statistics to test the joint significance of each of the other lagged endogenous variables in each equation of the model and also for joint significance of all other lagged endogenous variables in each equation of the models. The estimated results are given in Table 4. Here we present the results only for the equation dependent variable Aman and Boro only. From Table 4, we found that a chi-square (Wald) test statistics of for rainfall (RA) and humidity (HU) are 5.411010 and 6.014798 with reference to Aman represent the hypothesis that lagged coefficient of RA and $\mathrm{HU}$ in the regression equation of Aman are equal to zero which indicate that for Aman rice production, rainfall and humidity are granger cause for Rajshahi district. From this table we also found that all of the climatic variables are the influencing factors for aman rice production where rainfall and humidity have significant positive effect for aman production for the study area.

For the Boro rice, all of the climatic variables such as rainfalls, maximum temperature, minimum temperature, humidity, sunshine together are the influencing factor for the boro production where rainfall, minimum temperature and sunshine have significant positive effect/ granger cause for boro rice production where maximum temperature and humidity are not granger cause for boro rice production in case of Rajshahi district.

Table 4:-Estimation results for VAR Granger Causality/Block Exogeneity Wald Tests.

\begin{tabular}{|c|c|c|c|c|c|c|}
\hline Dependent variable & \multicolumn{3}{|c|}{ Aman } & \multicolumn{3}{c|}{ Boro } \\
\hline Excluded & Chi-square & df & P-Value & Chi-square & df & P-Value \\
\hline RA & 5.411020 & 2 & 0.0168 & 6.448670 & 2 & 0.0398 \\
\hline Tmax & 3.397207 & 2 & 0.1829 & 4.835030 & 2 & 0.1291 \\
\hline Tmin & 0.444497 & 2 & 0.8007 & 4.785436 & 2 & 0.0440 \\
\hline HU & 6.040798 & 2 & 0.0388 & 1.027247 & 2 & 0.5983 \\
\hline SS & 3.099763 & 2 & 0.2123 & 6.002208 & 2 & 0.0497 \\
\hline All & 9.849911 & 10 & 0.0438 & 20.06235 & 10 & 0.0287 \\
\hline
\end{tabular}

Impulse response function traces the effect of one time shock on one of the innovation on current and future values of endogenous variable. From Table 5, the impulse response function for aman rice up to 10 period indicates that the impulse response functions of the rainfall and Aman yield demonstrated that a unit shock in the rainfall will die out in the period four and remains negative till period two after that it becomes positive up to tenth period and effect rice production positively. From the impulse response function of the humidity will die out at period three and becomes negative and remain negative upto another three period and after that it becomes positive and effects the rice yield. The other variables such as maximum temperature, minimum temperature and sunshine also become positive after seventh and sixth period respectively.

Table 5:-Impulse Response Function for Aman rice.

\begin{tabular}{|c|c|c|c|c|c|c|}
\hline Period & Yield(Aman) & RA & TMAX & TMIN & HU & SS \\
\hline 1 & 118.4820 & 0.000000 & 0.000000 & 0.000000 & 0.000000 & 0.000000 \\
\hline 2 & 100.8367 & 25.19976 & -122.7399 & 71.96531 & -243.9233 & -44.41099 \\
\hline 3 & 400.0170 & 30.67725 & 417.8504 & -568.5190 & 442.4645 & 12.33573 \\
\hline 4 & 604.8437 & -1.125930 & -1417.923 & 1850.927 & -2108.108 & -152.3579 \\
\hline 5 & 3328.972 & -321.5081 & -5594.721 & -7354.253 & -7107.953 & 463.8174 \\
\hline 6 & 11214.76 & -863.1142 & -20393.31 & 27021.76 & -27128.56 & -1795.992 \\
\hline 7 & 43075.15 & 3588.245 & -76421.05 & -101116.1 & 100345.9 & 6516.676 \\
\hline 8 & 159206.0 & 13042.17 & 284290.9 & 376386.0 & 374653.1 & 24404.65 \\
\hline 9 & 594661.0 & 48938.71 & 1059853. & 1403093. & 1395273. & 90765.03 \\
\hline 10 & -2214510. & 182018.0 & 3948960. & 5228040. & 5200341. & -338366.2 \\
\hline
\end{tabular}

The impulse response function for Boro rice up to 10 periods indicates thatthe impulse response functions of the rainfall and Boro yield demonstrated that a unit shock in the rainfall becomes negative at second period and after that it becomes positive and positively affect the boro rice yield. Similarly, the series minimum temperature (Tmin) 
and sunshine becomes positive at period three and four respectively. Other series maximumtemperature (Tmax) becomes positive at period four and humidity becomes negative effect up to period ten.

Table 6:-Impulse Response Function for Boro rice.

\begin{tabular}{|c|c|c|c|c|c|c|}
\hline Period & Yield (Boro) & RA & TMAX & TMIN & HU & SS \\
\hline 1 & 146.0974 & 0.000000 & 0.000000 & 0.000000 & 0.000000 & 0.000000 \\
\hline 2 & 120.0922 & -72.24894 & -40.71400 & -15.46121 & -49.19854 & -86.66611 \\
\hline 3 & 126.4702 & 73.31653 & -16.70438 & 89.22608 & -83.18796 & -48.48297 \\
\hline 4 & 291.9131 & 614.7341 & 199.3891 & 392.1721 & -454.3587 & 40.75640 \\
\hline 5 & 992.1163 & 2453.316 & 1135.665 & 1267.253 & -1724.419 & 322.2852 \\
\hline 6 & 3352.205 & 8391.863 & 4177.917 & 4155.552 & -6014.144 & 1279.187 \\
\hline 7 & 11122.06 & 27925.61 & 14154.81 & 13679.41 & -20108.56 & 4460.799 \\
\hline 8 & 36821.89 & 92289.10 & 47074.07 & 45136.67 & -66647.60 & 15021.97 \\
\hline 9 & 121605.9 & 304455.1 & 155699.2 & 148779.4 & -220053.4 & 49815.11 \\
\hline 10 & 401169.4 & 1003699. & 513825.3 & 490339.7 & -725762.8 & 164519.8 \\
\hline
\end{tabular}

The variance decomposition analysis is typically performed by VAR models, which supplements impulse response function analysis (Ghatak, 1998). The intention of variance decomposition is to break up the variations on the endogenous variables in to component shocks to the VAR. From Table 7 and Figure 2, a variation of about 6 percent in period ten showed positive effect of rainfall for aman yield observed. For maximum temperature about $20 \%$ variation produced was in period ten in rice production. The highest variation due to humidity was approximately $36 \%$ in period ten showing positive effect of humidity on Aman rice production in Rajshahi district. Table 8 shows that about $11 \%$ variation of minimum temperature is observed at period ten and positive effect for boro rice production. The variation of sunshine is observed as 1.2 percent and highest variation is observed for rainfall (approximately 45\%) and positive effect on boro rice yield in case of Rajshahi district. The variance decomposition of boro rice due to rainfall, maximum temperature, minimum temperature, humidity and sunshine is also given in Figure 3.

Table 7:-Variance Decomposition for Aman rice.

\begin{tabular}{|c|c|c|c|c|c|c|c|}
\hline Period & S.E. & $\begin{array}{c}\text { Yield } \\
\text { (Aman) }\end{array}$ & RA & TMAX & TMIN & HU & SS \\
\hline 1 & 118.4820 & 100.0000 & 0.000000 & 0.000000 & 0.000000 & 0.000000 & 0.000000 \\
\hline 2 & 326.4292 & 22.71671 & 5.595957 & 14.13817 & 4.860360 & 55.83782 & 1.850985 \\
\hline 3 & 980.4338 & 19.16458 & 4.163966 & 19.73097 & 34.16309 & 26.55639 & 0.221015 \\
\hline 4 & 3351.247 & 4.897716 & 6.014045 & 19.59040 & 33.42864 & 41.84359 & 0.225606 \\
\hline 5 & 12591.28 & 7.337003 & 5.066194 & 21.13095 & 36.48243 & 34.83176 & 0.151674 \\
\hline 6 & 46586.51 & 6.331044 & 6.039161 & 20.70624 & 36.30898 & 36.45487 & 0.159704 \\
\hline 7 & 173824.8 & 6.595623 & 6.045426 & 20.81600 & 36.44700 & 35.94394 & 0.152021 \\
\hline 8 & 647441.1 & 6.522118 & 6.043853 & 20.78126 & 36.42323 & 36.07650 & 0.153042 \\
\hline 9 & 2412919. & 6.543273 & 6.044293 & 20.78943 & 36.43564 & 36.03484 & 0.152517 \\
\hline 10 & 8991334. & 6.537293 & 6.044171 & 20.78653 & 36.43278 & 36.04662 & 0.152604 \\
\hline
\end{tabular}

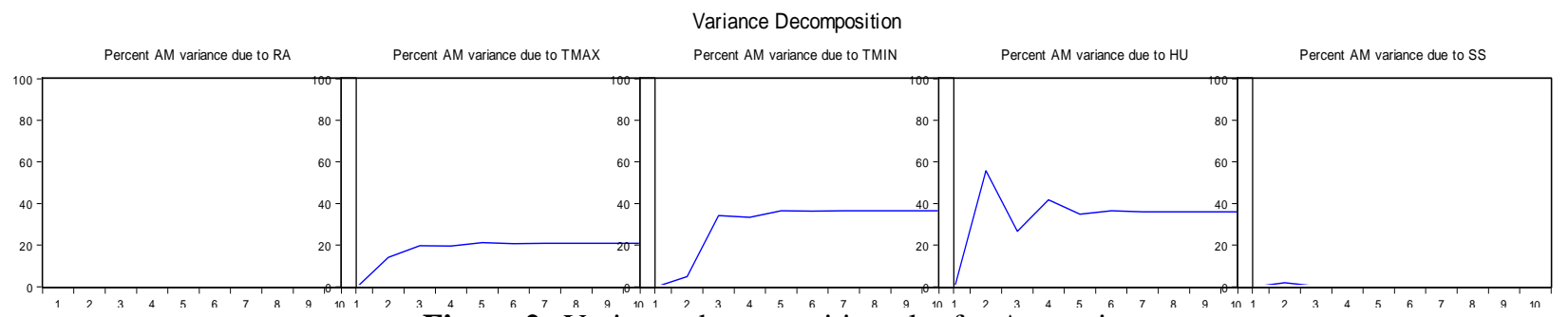

Figure 2:-Variance decomposition plot for Aman rice.

Table 8:-Variance Decomposition for Boro rice.

\begin{tabular}{|c|c|c|c|c|c|c|c|}
\hline Period & S.E. & $\begin{array}{c}\text { Yiled } \\
\text { (Boro) }\end{array}$ & RA & TMAX & TMIN & HU & SS \\
\hline 1 & 146.0974 & 100.0000 & 0.000000 & 0.000000 & 0.000000 & 0.000000 & 0.000000 \\
\hline
\end{tabular}




\begin{tabular}{|l|l|l|l|l|l|l|l|}
\hline 2 & 229.8145 & 67.72092 & 9.883441 & 3.138578 & 0.452618 & 4.582998 & 14.22145 \\
\hline 3 & 302.8133 & 56.44889 & 11.55474 & 2.112054 & 8.942978 & 10.18664 & 10.75470 \\
\hline 4 & 977.9903 & 14.32092 & 40.61761 & 4.359040 & 16.93729 & 22.56042 & 1.204717 \\
\hline 5 & 3732.650 & 8.047768 & 45.98726 & 9.556157 & 12.68909 & 22.89153 & 0.828200 \\
\hline 6 & 12966.23 & 7.350889 & 45.69900 & 11.17420 & 11.32297 & 23.41101 & 1.041921 \\
\hline 7 & 43398.17 & 7.224095 & 45.48529 & 11.63560 & 10.94630 & 23.55917 & 1.149539 \\
\hline 8 & 143796.3 & 7.215180 & 45.33432 & 11.77670 & 10.84993 & 23.62783 & 1.196041 \\
\hline 9 & 474806.2 & 7.221367 & 45.27434 & 11.83341 & 10.81383 & 23.64660 & 1.210453 \\
\hline 10 & 1565898. & 7.227326 & 45.24723 & 11.85519 & 10.79966 & 23.65545 & 1.215138 \\
\hline
\end{tabular}

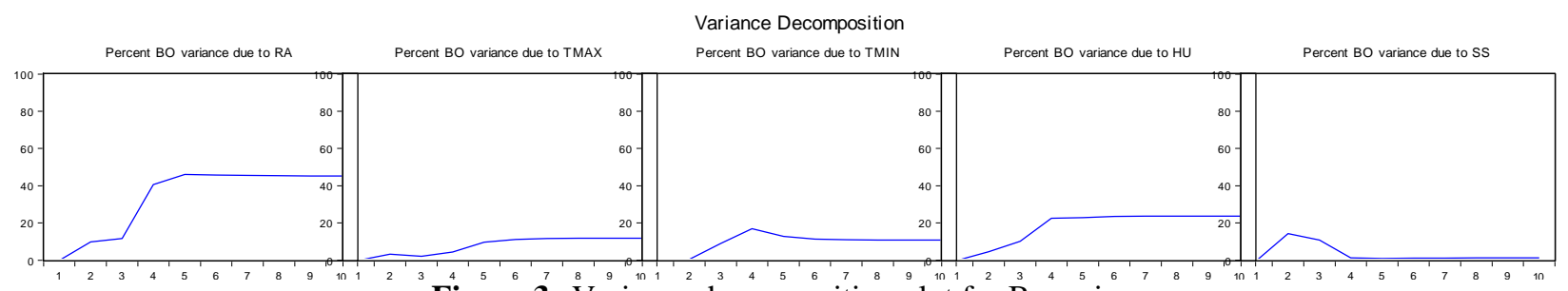

Figure 3:-Variance decomposition plot for Boro rice.

\section{Conclusion:-}

This paper investigates the impact of climatic variation on Aman and Boro rice production in Rajshahi district of Bangladesh by Vector Autoregression (VAR) model. The study used monthly data from 1987 to 2015 . The results of historical data estimation reveal that $89.6 \%$ variation can be explained by the climatic variables for aman rice and $96.1 \%$ variation can be explained by climatic variables for boro rice. So, the impacts of climatic variables are significant for both Aman and Boro rice. The result indicates that all of the climatic variables influence the Aman rice production where rainfall and humidity has significant positive effect. For Boro rice all of the climatic variables together influence the Boro rice production where rainfall, minimum temperature and sunshine has positive significant effect for Boro rice production in case of Rajshahi district.

\section{Acknowledgements:-}

This research was supported by University of Rajshahi research project (No. A 1047/5/52/RU/science-35/17-18)

\section{Reference:-}

1. Adejuwon, S.A. (2004). Impacts of climate variability and climate change on crop yield in Nigeria, Paper presented at Stakeholders' workshop on assessment of impacts and adaptation to climate change. ObafemiAwolowo University, I1e-Ife, Nigeria. p. 2-8.

2. Apata, T.G. (2010). Effects of global climate change on Nigerian agriculture: An empirical analysis. CBN J. Appl. Statistics, 2(1): 45-60.

3. BBS (Bangladesh Bureau of Statistics) (2014). Statistics and Informatics Division (SID), Ministry of Planning, Government of Bangladesh, Dhaka, Bangladesh. Available at: http:// www.bbs.gov.bd

4. Boubacar, I. (2010). The effects of drought on crop yields and yield variability in Sahel. The Southern Agricultural Economics Association Annual Meeting: The Southern Agricultural Economics Association, Orlando FL.

5. Basak, J.K., Ali, M.A., Islam, M.N., Rashid, M.A. (2010). Assessment of the effect of climate change on Boro rice production in Bangladesh using DSSAT model, Journal of Civil Engineering, 38, pp. 95-108.

6. Chang, C.C. (2002). The Potential Impact of Climate Change on Taiwan's Agriculture, Agricultural Economics, 27, 51-64. DOI: 10.1111/j.1574-0862.2002.tb00104.x

7. Chowdhury, I. U.A, and Khan, M. A. E.(2015). The impact of climate change on rice yield in Bangladesh: A timeseries analysis. RJOAS 4.40 (2015): 12-28.

8. Dickey, D.A. and. Fuller, W.A.(1979). Distribution of the Estimators for Autoregressive Time Series with a Unit Root, Journal of the American Statistical Association, 74, pp:427-431.

9. Dickey, D.A and Fuller, W.A.(1981). Likelihood Ratio Statistics for Autoregressive Time Series with a Unit Root, Econometrica, Vol. 49, pp. 1057-1072.

10. Deressa, T.T., and Hassan, R.M. (2009). Economic Impact of Climate Change on Crop Production in Ethiopia: Evidence from Cross-Section Measures, Journal of African Economics, 18, 529-554. DOI: 10.1093/jae/ejp002 
11. Farook, A. J. and Kannan, K. S. (2015). Climate change impact on rice yield in India-Vector AutoregressionApproch, Sri Lankan Journal of Applied Statistics, Vol (16-3), pp. 161-178.

12. Gbetibouo, G.A., Hassan, R.M. (2005). Measuring the Economic Impact of climate Change on Major South African Crops: A Ricardian Approach, Global and Planetary Change, 47, 143-152. DOI: 10.1016/j.gloplacha.2004.10.009.

13. Ghatak, A. (1998). Vector Autoregression Modelling and Forecasting Growth of South Korea, Journal of Applied Statistics, 25(5), 579-592. DOI: 10.1080/02664769822837

14. Granger, C.W.J. (1969). Investigating Causal Relations by Econometric Models and Cross Spectral Methods, Econometrica, 37, 424-438. DOI: 10.2307/1912791

15. Government of Bangladesh (GOB). Yearbook of Agricultural Statistics of Bangladesh; Ministry of Planning: Dhaka, Bangladesh, 2011.

16. Haim, D., Shechter, M., Berliner, P. (2008). Assessing the Impact of Climate Change on Representative Field Crops in Israel Agriculture: A Case Study of Wheat and Cotton, Climatic Change, 86, 425-440.DOI: 10.1007/s10584-007-9304-X

17. Hossain A, Teixeira da Silva J.A., Lozovskaya M.V. and Zvolinsky V.P. (2012). The effect of high temperature stress on the phenology, growth and yield of five wheat (Triticumaestivum L.) genotypes. The Asian and Australasian Journal of Plant Science and Biotechnology 6: 14-23.

18. Hossain, A., Teixeira da Silva, J.A. (2013). Phenology, Growth and Yield of Three Wheat (Triticum. aestivum L.) Varieties As Affected by High Temperature Stress. Not. Sci. Biol., 4, 97-106.

19. Holst, R., Yu, X., and Grun, C., (2010). Climate change, risk and grain production in China.AAEA, CAES, WAEA joint annual meeting. Agriculture and Applied Economics Association, Colorado.

20. Janjua, P. Z., Sanad,G., and Khan, N. U. (2010). Impact of climate change on wheat production: A case study of Pakistan, The Pakistan Development Review, 49:4 Part II (Winter 2010) pp. 799-822.

21. Kurukulasuriya, P., Ajwad, M.I. (2007). Application of the Ricardian Technique to estimate the impact of Climate Change on Small Holder Farming in Sri Lanka, Climatic Change, 81, 39-59. DOI: 10.1007/s10584005-9021-2

22. Kurukulasuriya, P. and R. Mendelsohn.(2008). A Ricardian analysis of the impact of climate change on African crop land.Afri. J. Agric. Resour. Econ. 2(1): 1-23.

23. Lansigan, F.P., de los Santos, W.L., Coladilla, J.O. (2000). Agronomic Impacts of Climate Variability on Rice Production in the Philippines.Agriculture, Ecosystems and Environment, 82, 129-137. DOI: 10.1016/s01678809(00)00222-X

24. Osterwald-Lenum, M.(1992). Practioners corner a note with quartiles of the asymptotic distribution of the maximum likelihood cointegration rank test statistics. Oxford Bulletin of Economics and Statistics, 54, pp: 461 472.

25. Phillips, P. C. B. and Perron, P. (1988). Testing for a Unit Root in Time Series Regression, Biometrika, Vol. 32, pp. 301-318

26. Rahman,M. M, Rahman, M.S., and Shah, M.A.R( 2018). Exploring the climate change effects on Boro rice yields of Rajshahi district in Bangladesh, International Journal of Advanced Research, 6(6), 748-755.

27. Rahman,M. M, Rahman, M.S., and Sultana, S. ( 2018). Impact of Climatic Parametters on Aman Rice production in Rangpur District, International Journal of Advanced Research, 6(8), 107-116.

28. Reilley, J., Watson, R., Zinyowera, M., Moss, R., and Dokken, D. (1996). Agriculture in a Changing Climate: Impacts and Adaptations. IPCC.

29. Sims, C.A. (1980). Macroeconomics and Reality, Econometrica, 48, 1-48. DOI: 10.2307/1912017.

30. Shakoor, U. Saboor, A., Baig, I., Afzal, A. and Rahman, A. (2015). Climate variability impacts on rice crop production in Pakistan, Pakistan J. Agric. Res. Vol.28, N0. 1, pp. 19-27.

31. Sarker, M.A.R., Alam, K., \&Gow, J. (2012). Exploring the relationship between climate change and rice yield in Bangladesh: An analysis of time series data, Agricultural Systems, 112, pp. 11-16.

32. Todd, R.M. (1984). Improving Economic Forecasting with Bayesian Vector Autoregression, Federal Reserve Bank of Minneapolis Quarterly Review, 18-29.

33. UNFCC (2007). The Fourth Assessment Report of the Intergovernmental Panel on Climate Change.https://unfccc.int/documents/5049\#beg.

34. World Bank (2013). 40turn down the heat: climate extremes, regional impacts, and the case for resilience, washington dc. 